\title{
NON-TRAUMATIC DIALYSIS IN THE YOUNG*
}

BY

\author{
MALCOLM E. CAMERON \\ Brisbane, Australia
}

\begin{abstract}
A DIALYSIS, or separation of the two ectodermal retinal layers at the ora, occurs in the following circumstances:
\end{abstract}

(1) In the senile eye, when the dialyses are usually small and multiple and situated anywhere in the periphery.

(2) In the aphakic eye, when the dialyses may be single or multiple, often in the upper half of the retina.

(3) In the normal emmetropic eye which has received definite trauma, either directly or indirectly, e.g. a non-penetrating blow from an air-gun pellet, or a fall from a horse. Here the dialysis is single and usually below and temporal, or above and nasal; or it may be situated at the point of the direct trauma.

(4) In the eyes of the young. Here most cases fall into the age group 15-35 years, and in this age group two types may be distinguished:

(a) The dialyses following rupture of the large bilateral symmetrical cysts situated below and temporally. These are quite rare.

(b) The single dialysis, almost invariably in the lower temporal region of the retina, and with no known cause.

This last type, which is also uncommon, will be discussed below by considering the non-traumatic cases that have occurred in the Brisbane General Hospital in the 11 years from July, 1947, to July, 1958.

\section{MATERIAL}

During this time, 22 cases of idiopathic dialysis were seen. (These include three cases where no diagram of the detachment was drawn, but where consideration of all the other findings leads one to believe a dialysis was present.) There were nine cases which followed, within a reasonable time, definite moderately severe trauma, only one case of dialysis associated with aphakia, and no cases of bilateral symmetrical cysts. These will not be discussed further.

HISTORY.-Only one-quarter of the patients consulted a doctor in the 2 weeks following interference with vision. One half of the patients waited for from between 1 and 6 months before consulting a doctor, and two boys, aged 11 and 15, waited 9 months and a year respectively before seeking treatment. (The loss of vision was mostly gradual rather than catastrophic, and this no doubt accounted for some of the delays in consultation.) Two patients, men aged 18 and 28, merely consulted

* Received for publication September 30, 1959. 
the ophthalmologist because of headaches, and the dialyses were discovered during the course of the routine examination.

The majority complained of blurred vision with the affected eye. Only five patients volunteered that the field of vision of one eye was affected, and in these it was the upper field that was involved. A small upper nasal loss of field is not easily perceived by the patient, because it is covered by the nasal field of the fellow eye. Even when the good eye is closed, loss of field in the affected eye is noticed only when the detachment has reached the equator.

Only one patient (with a giant dialysis to be discussed later) complained of spots floating in front of the eye, followed one month later by a film over the affected eye.

INCIDENCE.-Only 22 cases of idiopathic dialysis could be found in an 11-year period, in which the total new out-patient attendance at the Eye Clinic was approximately 50,000. The actual number of retinal detachment cases of all types was 184 , thus giving an incidence of idiopathic dialysis of 11 per cent.

SEX.-There were fifteen males (67 per cent.) and seven females (33 per cent.)

AGE.-Eighteen patients were in the 15- to 35-year age group. The youngest patient was a boy of 11 , the oldest a man of 58. The other two outside this age group were a girl of 14 and a man of 42 .

REFRACTION.-If, by emmetropia, one includes all cases with a refractive error of $1 \mathrm{D}$ or less, then there were only two ametropic eyes out of 22 , and these required $-1.5 \mathrm{D}$ cylinders.

SizE.- Of the nineteen cases accurately drawn, nine involved an area of retina of $30^{\circ}$ or less, another nine involved an area of $30^{\circ}-90^{\circ}$, and in one patient, a male timber hauler of 24 , a dialysis a little greater than $180^{\circ}$ was seen. This last occurred in the upper outer part of the right eye. The retina had fallen down over itself; this is shown in Fig. 1 (opposite) by the area A B C. Medially in this area the retina was grossly ballooned, while laterally it was shallower. The area above A B was seen to comprise choroid only, with no retinal architecture at all. The only normal strip of retina was seen to occupy the periphery of the lower nasal quadrant (below A C). The visual field was correspondingly reduced to a small part of the upper temporal field (Fig. 2, opposite). No surgical treatment was attempted.

Situation.-In twelve out of the nineteen cases, the dialysis was situated in the lower temporal quadrant; in five directly below; and in two above.

SIDE.-The right and left eyes were equally involved, and in no case did another dialysis occur in the fellow eye.

OPERATION RESULTS.-In one-third of the cases operated on, the visual acuity was restored to $6 / 9$ or better; these were mostly operated on within 2 weeks of the occurrence. This correlation is not absolute, as the post-operative visual acuity depends on whether the detachment has involved the macula, rather than on the time involved, although these are in general directly related.

Sometimes the detachment was self-limiting, as in a female of 24 with a 4 months' history and "high-water marks"; but in this case the macula had been involved and the post-operative visual acuity was only $6 / 60$. 
FIG. 1.-Partly diagrammatic sketch of right fundus of a timber-hauler aged 24 .

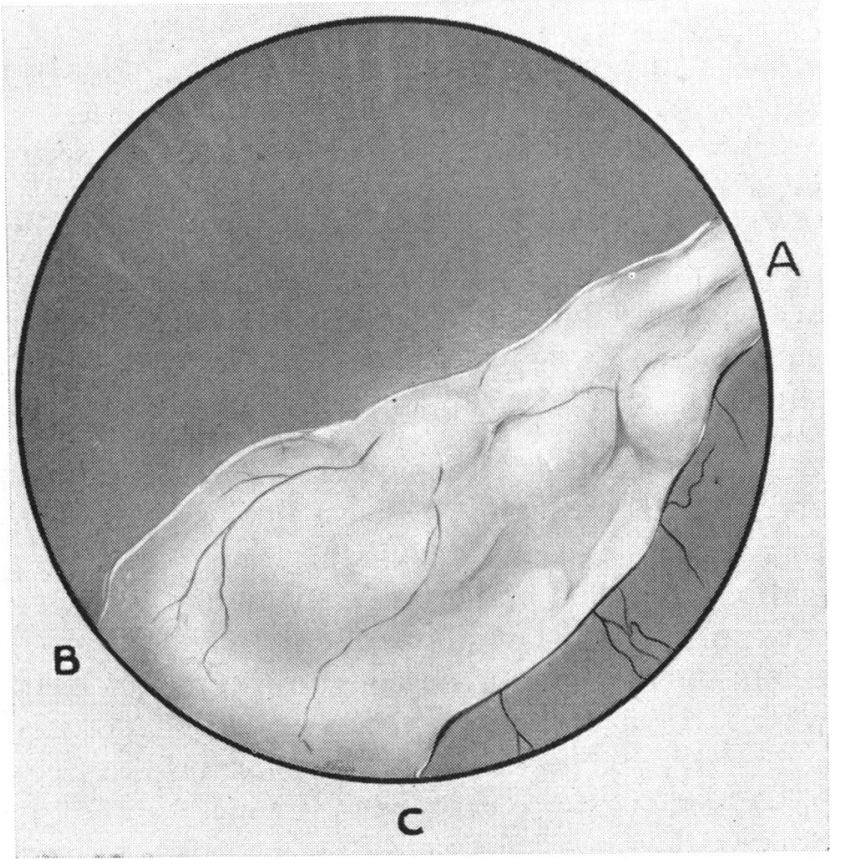

TARGET 330 WHITE

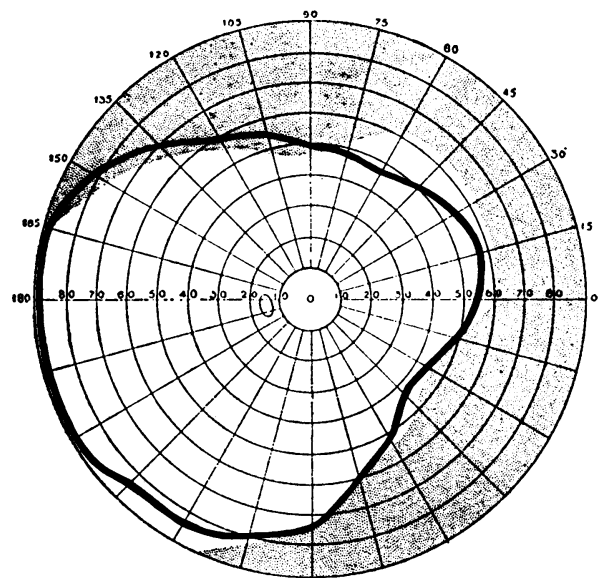

LEFT EYE

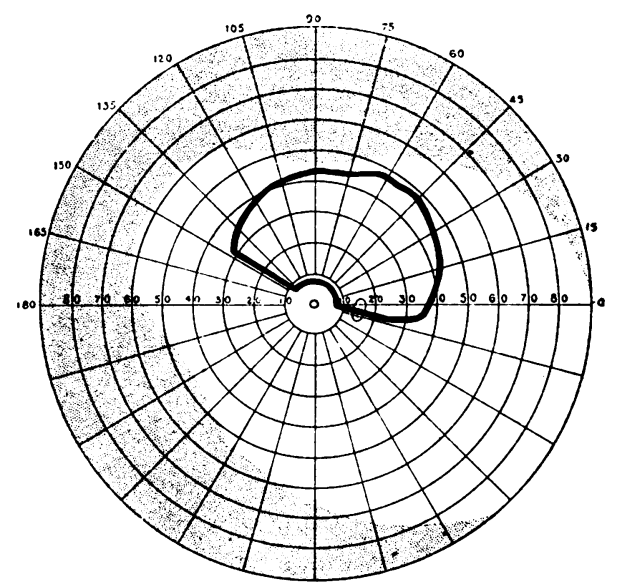

RIGHT EYE

FIG. 2.-Peripheral fields of same patient.

The standard operation performed in all cases was the classical diathermy and evacuation, and in every case in which adequate post-operative notes were available, it was successful. Two operations were performed in consecutive months on each of four patients, because of non-attachment after the first operation, and in all these a final successful result was obtained. 


\section{Aetiology}

The cause of non-traumatic dialysis in this young age group is rather baffling, and two factors require consideration:

(1) Degenerative processes in the extreme peripheral part of the retina, i.e. at the ora serrata;

(2) Internal forces such as accommodation (Teng and Katzin, 1953), normal movements of the eye in association with abnormal vitreous adhesions at the ora (Gonin, 1906), and the forces of differential growth, in which the pars plana, growing forward at a faster rate than the peripheral retina, stretches the latter, snapping one or several oral bays.

Perhaps degenerative processes may be enough in themselves to bring about detachment, but it is just in this young age group that degenerative changes are least and dialysis most common. In a recent study of 24 eyes with well-marked oral cysts occurring in the age group 44 to 78 years, there was not one case of idiopathic retinal dialysis (Grignolo, Schepens, and Heath, 1957). Cystoid degeneration at the ora over the age of 50 is universal, yet dialysis at this age is very rare, and Leffertstra (1948) goes so far as to state that in 90 per cent. of cases the cysts are secondary to the dialysis.

The only well-proven type of degeneration leading to dialysis is the large, bilateral symmetrical cyst. This is thought to begin by the appearance of a small cyst between the outer nuclear and outer molecular layers. This grows in size or coalesces with others, until a large cyst has been formed. The inner wall (next to the vitreous) consists of degenerated Mullerian fibres; the outer wall consists of the remains of the rods and cones (Teng and Katzin, 1953).

The only case in the present series resembling the above was that of a single woman aged 21, a trainee nurse. When admitted to hospital on July 20, 1953, she gave a history that 8 days previously the vision in the left eye became blurred, and this was followed by severe impairment 5 days after. There was found to be a detachment involving the lower half of the retina, with a cyst the size of the disc at 6 o'clock and a small haemorrhage nearby. No dialysis could be seen despite intensive searching during the next month. Two weeks after diathermy and evacuation on August 24, 1953, the cyst and retina were not altered in appearance, but a $15^{\circ}$ dialysis could be seen below it. After further diathermy and evacuation on September 17, 1953, the cyst was seen to have shrunk considerably, and a haemorrhage was present in it. The retina became re-attached, but the best vision was only $6 / 24$. In reply to a recent questionnaire (July, 1959), she said there had been no involvement subsequently of the normal right eye (emmetropic 6/5) and that the visual acuity in the left eye had remained the same.

The initial cystic area which enlarges may be produced by the diminution of the blood supply of either the choriocapillaris or the peripheral retinal circulation. At the periphery of the retina, there is only one wide-mesh capillary network in contradistinction to the two-, three-, or four-layer 
net-work present in other parts of the retina. Immediately posterior to the ora, there is a $1-\mathrm{mm}$. band of retina without blood vessels (Michaelson, 1954).

Weve (1935) inquired into the frequency of birth trauma in cases of dialysis but could find no correlation. In the present series, a questionnaire was sent to seventeen former patients, to see if there was any connexion between dialysis and prematurity, but none was found in the twelve who replied.

With regard to causative internal forces, Teng and Katzin (1953) believe that accommodation plays a large part in the creation of cysts and holes at the ora. However, if this were so, one would expect a greater incidence of dialysis in hypermetropia but, as shown above, dialysis occurs characteristically in emmetropic young adults. It is thus seen that no explanation so far - considered is in accordance with the observed facts.

Because of this, the author puts forward the following theoretical explanation mentioned previously-that differential increased growth of the pars plana, as compared with that of the peripheral retina, exerts anterior traction on the latter, weakening it and eventually snapping it at the ora. The ora represents the point at which the loose separation between the neural and pigment epithelium changes to the firm adhesion of the pars plana. An example of such differential growth is seen in the asymmetry of the ora, the nasal ora being $1 \mathrm{~mm}$. closer to the limbus than the temporal, due to postnatal temporal growth (Wolff, 1940). Also, the more frequent occurrence of retinal detachment in myopic eyes is thought to be caused by the stretching of the retina, due to its inability to keep up with the growth of the outer coats of the eyeball. It is thought then that the normal greater post-natal temporal growth becomes accentuated in cases of idiopathic dialysis, so that the temporal oral region is stretched and weakened. This would help explain the characteristic temporal situation, not accounted for by any other theory.

Another factor which may be contributory, is that there is a decreased blood supply to this part of the retina, which is phylogenetically and ontogenetically the oldest part, and the last to become vascularized (Weve, 1953).

All the above cases were examined by the direct method of ophthalmoscopy, helped on rare occasions by scleral indentation. The indirect method is used by the author mainly in moderate or high myopia, or where the dialysis is difficult to see because of opacities of the media. In view of the controversy over the two methods, six patients previously operated on for retinal dialysis were examined directly with a focusing and a non-focusing ophthalmoscope, and indirectly with the Schepens binocular ophthalmoscope. In both methods, the patient was seated with the affected eye turned maximally in the required direction, and was observed after maximum mydriasis had been obtained with 2 per cent. homatropine and cocaine and 10 per cent. neosynephrine.

With direct observation, the surgeon bent over above the patient, shining the light down obliquely; with the indirect method, he sat facing the patient. 
Because of previous diathermy, landmarks were easy to recognize. The original dialysis was often seen with the retina re-attached to its new resting place. It was found that more of the anterior retina could be seen with the focusing direct ophthalmoscope than with either the non-focusing direct model or the Schepens ophthalmoscope. The area of view is not so great, nor is the retina so clearly seen with the direct method, because of the obliquity of the light, but this is more than compensated for by the ease of manipulation and the greater extent of anterior retina which can be seen. It is possible that more of the anterior retina would have been seen with the Schepens model had the patient been lying down (Schepens and Bahn, 1950), but this was not done in the present series.

There was only one case of almost certain dialysis, in a male aged 34 years, in which the records show that the surgeon (using the direct method) searched for the tear but could not find it. A grey area was seen in the extreme periphery at half past 4 o'clock in the left eye, but the retina peripheral to this area could not be seen. However, diathermy at this suspicious area produced re-attachment.

It would thus seem reasonable for those of us more skilled in the direct method of ophthalmoscopy to continue with it, bearing in mind that a focusing direct ophthalmoscope will enable one to see further out in the periphery than a non-focusing direct ophthalmoscope. The value of scleral indentation at the ora or pars plana in both direct and indirect methods should also be remembered. Where no dialysis can be seen with the direct method, even with scleral indentation, or if for any reason the examiner is not satisfied, indirect binocular ophthalmoscopy should be used, with the patient supine.

\section{SUMMARY}

(1) 22 cases of idiopathic retinal dialysis are reviewed.

(2) A new theory is advanced to explain their occurrence.

(3) Conventional diathermy has been found to give excellent results in this type of detachment.

(4) Observation by a focusing direct ophthalmoscope is considered to be quite satisfactory in these cases.

I wish to thank Dr. A. D. D. Pye for permission to investigate the Brisbane General Hospital records; my colleagues for permission to examine their cases; and Miss L. Pegus for the fundus drawing.

\section{REFERENCES}

GoNIN, J. (1906). In “Encyclopédie française d'ophtalmologie”, p. 691. (Quoted by Weve, 1953.) Grignolo, A., SChePens, C. L., and HeATH, P. (1957). A.M.A. Arch. Ophthal., 58, 530.

LefFERTSTRA, L. J. (1948). "Over Orascheuren”. Thesis, Utrecht (Quoted by Weve, 1953).

MichaELSON, I. C. (1954). " "Retinal Circulation in Man and Animals". Thomas, Springfield, Ill. SCHEPENS, C. L., and BAHN, G. C. (1950). Arch. Ophthal. (Chicago), 44, 677.

Teng, C. C., and Katzin, H. M. (1953). Amer. J. Ophthal., 36, 29.

WEVE, H. (1935). Arch. Augenheilk., 109, 49.

WEV (1953). Trans. ophthal. Soc. Aust., 13, 35.

WolfF, E. (1940). "The Anatomy of the Eye and Orbit", 3rd ed., fig. 34, p. 48. Lewis, London. 